\title{
Análise de indicadores assistenciais em uma Unidade de Terapia Intensiva Pediátrica na cidade de Fortaleza/CE
}

\author{
Analysis of social indicators in a Pediatric Intensive Care \\ Unit in the city of Fortaleza/CE
}

\author{
Carlos Ariel Souza de Oliveira ${ }^{1}$, Francisco Cid Coelho Pinto', \\ Thiago Brasileiro de Vasconcelos ${ }^{1}$, Vasco Pinheiro Diógenes Bastos ${ }^{1}$
}

\begin{abstract}
Resumo
Introdução: Os indicadores de saúde são medidas que refletem informações sobre diferentes dimensões e atributos da saúde e dos fatores que a determinam. O estudo teve como objetivo analisar os indicadores de uma Unidade de Terapia Intensiva Pediátrica, traçando o perfil do diagnóstico dos pacientes internados na UTI em estudo e verificando as taxas de ocupação, tempo de permanência e outros dados importantes para o hospital. Métodos: A pesquisa foi de caráter descritivo, exploratório, longitudinal, documental, prospectivo e de abordagem quantitativa. A coleta de dados foi realizada de Março de 2015 a Fevereiro de 2016, conforme aprovação do Comitê de Ética do referido hospital. Resultados: A amostra em estudo tinha como idade média 10,63 \pm 0,50 anos, com 73,10 $\pm 2,45 \%$ de prevalência do gênero masculino, onde o Traumatismo Crânio Encefálico (TCE) sobressaiu-se como causa, e a taxa de ocupação no período do estudo foi de $86,13 \%$. Conclusão: Concluiu-se que o gênero masculino teve uma prevalência na UTI Pediátrica em relação ao gênero feminino, apresentando como acometimento principal Traumatismo Crânio Encefálico, evidenciando uma média de 8,72 \pm 0,47 dias de utilização da ventilação mecânica e 75,30\% de taxa de utilização de ventilação mecânica.
\end{abstract}

Palavras-chave: indicadores; pediatria; terapia intensiva; sistema de saúde.

\begin{abstract}
Introduction: health indicators are measures that provide information on different health dimensions and attributes and on the factors that determine them. This study aimed to analyze the indicators of a Pediatric intensive care unit through the diagnostic of the profile of patients admitted to the ICU and by checking the occupancy rates, length of stay and other important data to the hospital. Methods: the research has a descriptive, exploratory, longitudinal, prospective, documentary and quantitative approach. Data collection was carried out between March 2015 and February 2016, after approval from the hospital Ethics Committee. Results: the sample studied had the average age of $10.63 \pm 0.50$ years with $73.10 \pm 2.45 \%$ of male prevalence, and Traumatic Brain Injury (TBI) stood out as the cause of admission. The occupancy rate during the studied period was $86.13 \%$. Conclusion: the results led to the conclusion that the males predominate in Pediatric Intensive Care Unit in relation to females, with Traumatic Brain Injury as the main cause and the average of $8.72 \pm 0.47$ days of mechanical ventilation use in $75.30 \%$ of the cases.
\end{abstract}

Keywords: indicators; pediatrics; intensive care; health system. 


\section{INTRODUÇÃO}

Os indicadores de saúde, em termos amplos, são medidas que refletem, indiretamente, relevantes informações sobre diferentes dimensões e atributos da saúde e dos fatores que a determinam, incluindo o desempenho do sistema de saúde ${ }^{1}$.

A qualidade assistencial compartilhada entre os profissionais da área da saúde, sobretudo os que atuam nas esferas gerenciais, vem sendo cada vez mais discutida, tendo em vista as exigências dos usuários dos estabelecimentos de saúde, quanto ao maior comprometimento destas instituições. Sendo assim, os indicadores têm como objetivo auxiliar o monitoramento dos possíveis erros e eventos adversos ocorridos durante internações em hospitais ${ }^{2}$.

Os indicadores servem de instrumento para qualificação, avaliação e monitoração da saúde e seus determinantes, seja em relação a uma população, pessoa ou mesmo uma instituição ${ }^{3}$. Estes indicadores são instrumentos valiosos para auxiliar os hospitais a identificar áreas que precisam ser mais bem estudadas, com visão voltada à melhoria da qualidade dos cuidados prestados aos pacientes. A Unidade de Terapia Intensiva (UTI) faz parte da classificação dos indicadores de segurança ${ }^{4}$.

A qualidade da assistência em UTI pode ser avaliada através de indicadores que, mediante a utilização de parâmetros concretos, tem por finalidade uma demonstração numérica do desempenho técnico e de processos de tratamento desenvolvidos nessa Unidade. Por esse motivo, os indicadores que serão empregados devem ser concebidos cuidadosamente para que sejam completos, práticos, robustos e válidos, e com isso poderemos nos concentrar nas áreas que exigem uma maior investigação $0^{5}$.

De acordo com a Agência Nacional de Vigilância Sanitária (ANVISA), em sua Instrução Normativa ${ }^{\circ} 4$, que disserta a respeito dos indicadores para avaliação de Unidades de Terapia Intensiva, em consonância com art. 48 da RDC/ANIVA no 7 , destaca-se que o monitoramento dos respectivos indicadores devem ser mensais, dentre os quais podem ser destacados: Tempo de permanência na Unidade de Terapia Intensiva; Incidência de Pneumonia Associada à Ventilação Mecânica (PAV); Taxa de utilização de Ventilação Mecânica (VM) ${ }^{6}$.

$\mathrm{O}$ estudo teve como objetivo geral analisar os indicadores de uma Unidade de Terapia Intensiva Pediátrica, e específicos: Traçar o perfil do diagnóstico dos pacientes internados na UTI em estudo; Verificar as taxa de ocupação e tempo de permanência na referida Unidade; Verificar a taxa de desmame com sucesso na Unidade em estudo; bem como detectar o uso da Ventilação Mecânica prolongada.

\section{METODOLOGIA}

A pesquisa foi de caráter descritivo, exploratório, longitudinal, documental, prospectivo e de abordagem quantitativa, realizada no Hospital Instituto Doutor José Frota (IJF), situado na Rua Barão do Rio Branco, S/N, Centro, Fortaleza, CE.
O IJF é o maior hospital terciário de urgência e emergência do Ceará, com atendimento 24 horas para pacientes de alta complexidade, apresentando uma média de 13 mil atendimentos por mês, em que cerca de $50 \%$ das pessoas atendidas são de municípios do interior e região metropolitana de Fortaleza. Referência em todo o Brasil para o tratamento de queimaduras e intoxicações, o IJF tem um Centro de Tratamento de Queimados (CTQ) e um Centro de Assistência Toxicológica (CEATOX), entre outros serviços.

A Unidade de Terapia Intensiva (UTI) do Instituto Doutor José Frota é constituída por 33 leitos, que são subdivididos em 7 leitos para UTI Pediátrica e 26 leitos para UTI Adulta.

A coleta de dados foi realizada de março/15 a fevereiro/16, conforme aprovação do Comitê de Ética em Pesquisa do Instituto Dr. José Frota (Protocolo ${ }^{\circ}$ 1.177.954).

A amostra foi constituída dos prontuários de crianças internada na Unidade de Terapia Intensiva Pediátrica do referido hospital no período do estudo, independentemente do gênero ou do quadro patológico em que se encontravam. Nenhum prontuário foi excluído, tendo em vista que a amostra foi igual à população do estudo.

Os dados foram registrados em um instrumento de coleta de dados, contendo tópicos como: idade, gênero, motivo de ingresso na UTI, dias que se utilizou de suporte ventilatório e taxa de ocupação da UTI Pediátrica. O item motivo de internação foi definido pelo diagnóstico que levou à admissão no hospital, sendo agrupados em: TCE (Traumatismo Crânio Encefálico) por qualquer motivo; vítima de PAF (Projétil por Arma de Fogo) em qualquer parte do corpo; TCE+PAF para aqueles em que o TCE foi em decorrência de PAF; e Outros, para os diversos motivos para admissão, como: intoxicação exógena, queimaduras, cirurgias abdominais e torácicas, traumatismo raquimedular, broncoaspiração e outros.

Os dados obtidos foram analisados no software Statistical Package for Social Sciences (SPSS) versão 20.0, e os resultados apresentados em forma de gráficos e tabelas.

O estudo seguiu os aspectos éticos que envolvem a pesquisa com seres humanos, como garantia da confidencialidade, do anonimato, da não utilização das informações em prejuízo dos indivíduos e do emprego das informações somente para os fins previstos na pesquisa.

\section{RESULTADOS}

A UTI Pediátrica do Instituto Dr. José Frota recebe crianças/adolescentes com idade inferior a 18 anos, e no período do estudo a média de idade foi de $10,63 \pm 0,50$ anos. O mês de novembro/15 apresentou uma média de idade elevada, quando comparada com os outros meses analisados, porém sem diferença estatística significante $(\rho=0,082)$ (Quadro 1$)$. 
Com relação ao gênero dos pacientes internados na UTI em estudo, foi possível evidenciar que o gênero masculino apresentava um percentual médio no período de $73,10 \pm 2,45 \%$, e o feminino $26,90 \pm 8,48 \%$. Entretanto, essa diferença foi mais acentuada no mês de dezembro/15, porém sempre o gênero masculino era preponderante (Quadro 1).

A taxa de ocupação no período do estudo apresentou-se no primeiro, no quarto e no décimo primeiro mês inferior a $80 \%$, porém nos outros meses analisados ficou acima de $85 \%$, com uma média percentual de ocupação nesse período de $86,13 \pm 3,17 \%$. Essa taxa de ocupação média representa em leitos ocupados $6,12 \pm 0,19$, sendo o mês de março/15 com 4,32 $\pm 0,20$ leitos ocupados por dia, apresentando a menor taxa do período, e a

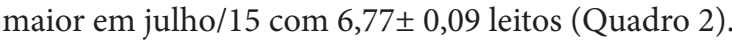

$\mathrm{Na}$ pesquisa, os pacientes na Unidade de Terapia Intensiva Pediátrica apresentaram um tempo de permanência média de $15,52 \pm 0,94$ dias. No mês de junho/15, apresentou o menor

Quadro 1. Distribuição dos dados de acordo com a idade e o gênero dos pacientes internados na UTI Pediátrica no período de março/2015 a fevereiro/2016. Fortaleza/CE, 2016

\begin{tabular}{|l|c|c|c|}
\hline \multirow{2}{*}{\multicolumn{1}{c|}{ Meses }} & Idade Média & \multicolumn{2}{c|}{ Gênero (\%) } \\
\cline { 3 - 4 } & (Anos) & Masculino & Feminino \\
\hline Março/15 & $12,78 \pm 3,93$ & 71,43 & 28,57 \\
\hline Abril/15 & $10,18 \pm 6,17$ & 75,00 & 25,00 \\
\hline Maio/15 & $10,12 \pm 6,00$ & 68,42 & 31,58 \\
\hline Junho/15 & $11,09 \pm 5,61$ & 66,67 & 33,33 \\
\hline Julho/15 & $8,49 \pm 6,48$ & 68,42 & 31,58 \\
\hline Agosto/15 & $8,89 \pm 6,06$ & 81,25 & 18,75 \\
\hline Setembro/15 & $8,77 \pm 4,88$ & 80,00 & 20,00 \\
\hline Outubro/15 & $12,84 \pm 3,71$ & 52,63 & 47,37 \\
\hline Novembro/15 & $13,12 \pm 3,10$ & 68,75 & 31,25 \\
\hline Dezembro/15 & $10,26 \pm 5,02$ & 83,33 & 16,67 \\
\hline Janeiro/16 & $8,53 \pm 5,08$ & 80,00 & 20,00 \\
\hline Fevereiro/16 & $12,47 \pm 4,46$ & 81,25 & 18,75 \\
\hline
\end{tabular}

Quadro 2. Distribuição dos dados de acordo com o número de Leitos/ Dia e a Taxa de Ocupação na UTI Pediátrica no período de março/2015 a fevereiro/2016. Fortaleza/CE, 2016

\begin{tabular}{|l|c|c|}
\hline \multicolumn{1}{|c|}{ Meses } & $\begin{array}{c}\text { Leitos } \\
\text { (Dias) }\end{array}$ & $\begin{array}{c}\text { Taxa de Ocupação } \\
\text { (\%) }\end{array}$ \\
\hline Março/15 & 4,32 & 61,75 \\
\hline Abril/15 & 6,40 & 91,42 \\
\hline Maio/15 & 6,23 & 88,94 \\
\hline Junho/15 & 5,97 & 66,29 \\
\hline Julho/15 & 6,77 & 96,77 \\
\hline Agosto/15 & 6,33 & 90,32 \\
\hline Setembro/15 & 6,66 & 95,23 \\
\hline Outubro/15 & 5,86 & 85,71 \\
\hline Novembro/15 & 6,60 & 94,28 \\
\hline Dezembro/15 & 6,68 & 95,39 \\
\hline Janeiro/16 & 5,52 & 78,80 \\
\hline Fevereiro/16 & 6,21 & 88,66 \\
\hline
\end{tabular}

tempo permanência (9,42 dias), ficando o mês novembro/15 com o maior (19,80 dias) (Quadro 3).

Ao ser analisado o diagnóstico que levou à internação dessa população, foi possível detectar que o Traumatismo Crânioencefálico (TCE), provocado por traumas (quedas, espancamento, acidentes automotores), apresentou uma preponderância em todos os meses analisados, destacando o mês de abril, em que existiu uma associação com PAF (Projétil por Arma de Fogo), e nos meses de outubro e novembro de 2015 ocorreu a prevalência de outros diagnósticos (Quadro 3).

Ao relacionar o tempo de permanência na UTI Pediátrica com o diagnóstico de internação, é evidente a presença de pacientes vítimas de TCE, porém no mês de novembro/15, quando ocorreu o maior tempo médio de permanência, a presença de outros diagnósticos apresentou um maior percentual. Evidencia-se que o menor tempo de permanência foi em junho/15, mês esse em que houve um equilíbrio entre os diagnósticos de TCE e de TCE associado a PAF (Quadro 3).

Com relação à utilização da Ventilação Mecânica na UTI Pediátrica, foi evidenciado que nos últimos três meses analisados ocorreu um aumento nesse tempo médio de utilização da ventilação, sendo que nesse período do estudo a média de dias de uso ficou em 8,93 \pm 0,78 dias, abaixo desses meses (Quadro 4).

$\mathrm{Na}$ pesquisa foi evidenciado que o tempo de permanência na UTI Pediátrica foi menor no mês de junho/15 e também se apresentou o menor tempo de utilização de ventilação mecânica. Ao analisar a maior taxa de uso de ventilação mecânica, presente no mês de novembro/15, que foi também o mês que teve o maior tempo de permanência na UTI Pediátrica, porém, essa proporcionalidade não se mantém presente na correlação dos outros dados, não apresentando relação estatisticamente significativa (Quadro 4).

No Quadro 2 foi possível evidenciar que não existe uma relação da Taxa de Utilização da Ventilação Mecânica com o Tempo de Utilização da Ventilação Mecânica, muito menos com o Tempo de Permanência na Internação na UTI Pediátrica (Quadro 4).

Quando analisada a Taxa de Sucesso do Desmame da Ventilação Mecânica na UTI Pediátrica, foi possível detectar que nos meses de junho/15 a agosto/15 essa taxa foi abaixo $50 \%$, assim como nos meses de novembro/15 e janeiro/16, porém no período do estudo a taxa média de sucesso ficou em $53,10 \pm 3,98 \%$ (Figura 1).

A utilização por tempo prolongado da Ventilação Mecânica na UTI Pediátrica foi acentuada no mês de julho/15, ficando com $25 \%$ dos pacientes que usavam o suporte ventilatório por mais de 21 dias. No entanto, notou-se uma baixa tanto no mês de março quanto no de outubro/15, evidenciando uma ausência da taxa de pacientes em ventilação mecânica (Figura 2). 
Quadro 3. Distribuição dos dados de acordo com o Tempo de Permanência e do Diagnóstico de Internação na UTI Pediátrica no período março/2015 a fevereiro/2016. Fortaleza/CE, 2016

\begin{tabular}{|c|c|c|c|c|c|}
\hline \multirow{2}{*}{ Meses } & \multirow{2}{*}{$\begin{array}{c}\text { Tempo de } \\
\text { Permanência (dias) }\end{array}$} & \multicolumn{4}{|c|}{ Diagnóstico da Internação (\%) } \\
\hline & & TCE & PAF & TCE+PAF & OUTROS \\
\hline Março/15 & 12,18 & 43,28 & 7,46 & 38,80 & 10,44 \\
\hline Abril/15 & 17,45 & 41,66 & 0,00 & 44,27 & 14,06 \\
\hline Maio/15 & 16,08 & 66,32 & 0,00 & 22,27 & 11,39 \\
\hline Junho/15 & 9,42 & 34,07 & 16,20 & 32,40 & 17,31 \\
\hline Julho/15 & 17,50 & 54,76 & 1,90 & 10,00 & 33,33 \\
\hline Agosto/15 & 19,60 & 53,06 & 6,12 & 14,28 & 36,53 \\
\hline Setembro/15 & 13,33 & 46,00 & 13,00 & 0,00 & 41,00 \\
\hline Outubro/15 & 14,30 & 25,27 & 15,05 & 6,98 & 56,45 \\
\hline Novembro/15 & 19,80 & 40,90 & 16,16 & 0,00 & 43,43 \\
\hline Dezembro/15 & 18,81 & 45,51 & 22,70 & 4,83 & 29,85 \\
\hline Janeiro/16 & 11,40 & 40,70 & 20,46 & 7,60 & 21,63 \\
\hline Fevereiro/16 & 16,36 & 61,66 & 7,46 & 8,33 & 15,55 \\
\hline
\end{tabular}

Quadro 4. Distribuição dos dados de acordo com o Tempo de Permanência de Internação, Tempo e Taxa de Utilização da Ventilação Mecânica na UTI Pediátrica no período março/2015 a fevereiro/2016. Fortaleza/CE, 2016

\begin{tabular}{|l|c|c|c|}
\hline \multicolumn{1}{|c|}{ Meses } & $\begin{array}{c}\text { Tempo de Permanência } \\
\text { (dias) }\end{array}$ & $\begin{array}{c}\text { Tempo de Utilização da VM } \\
\text { (dias) }\end{array}$ & $\begin{array}{c}\text { Taxa de Utilização da VM } \\
\text { (\%) }\end{array}$ \\
\hline Março/15 & 12,18 & 9,00 & 60,44 \\
\hline Abril/15 & 17,45 & 7,21 & 58,85 \\
\hline Maio/15 & 16,08 & 8,63 & 84,21 \\
\hline Junho/15 & 9,42 & 5,42 & 79,16 \\
\hline Julho/15 & 17,50 & 10,75 & 84,21 \\
\hline Agosto/15 & 19,60 & 11,25 & 64,28 \\
\hline Setembro/15 & 13,33 & 10,27 & 73,33 \\
\hline Outubro/15 & 14,30 & 8,36 & 73,68 \\
\hline Novembro/15 & 19,80 & 9,79 & 87,50 \\
\hline Dezembro/15 & 18,81 & 8,81 & 84,21 \\
\hline Janeiro/16 & 11,40 & 6,59 & 80,00 \\
\hline Fevereiro/16 & 16,36 & 8,50 & 73,68 \\
\hline
\end{tabular}

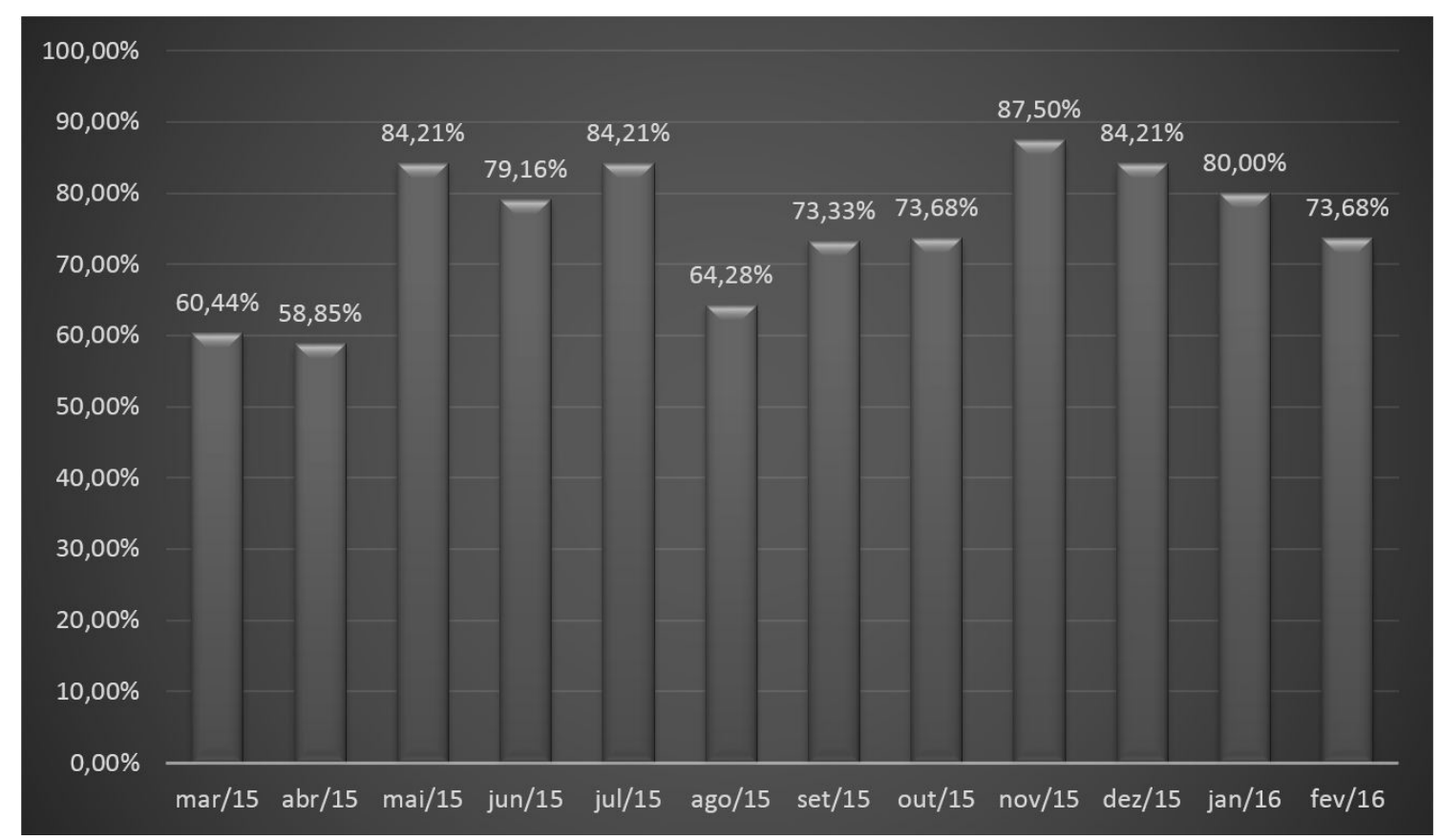

Figura 1. Distribuição dos dados de acordo com a Taxa de Sucesso de Desmame da Ventilação Mecânica na UTI Pediátrica no período de março/2015 a fevereiro/2016. Fortaleza/CE, 2016 


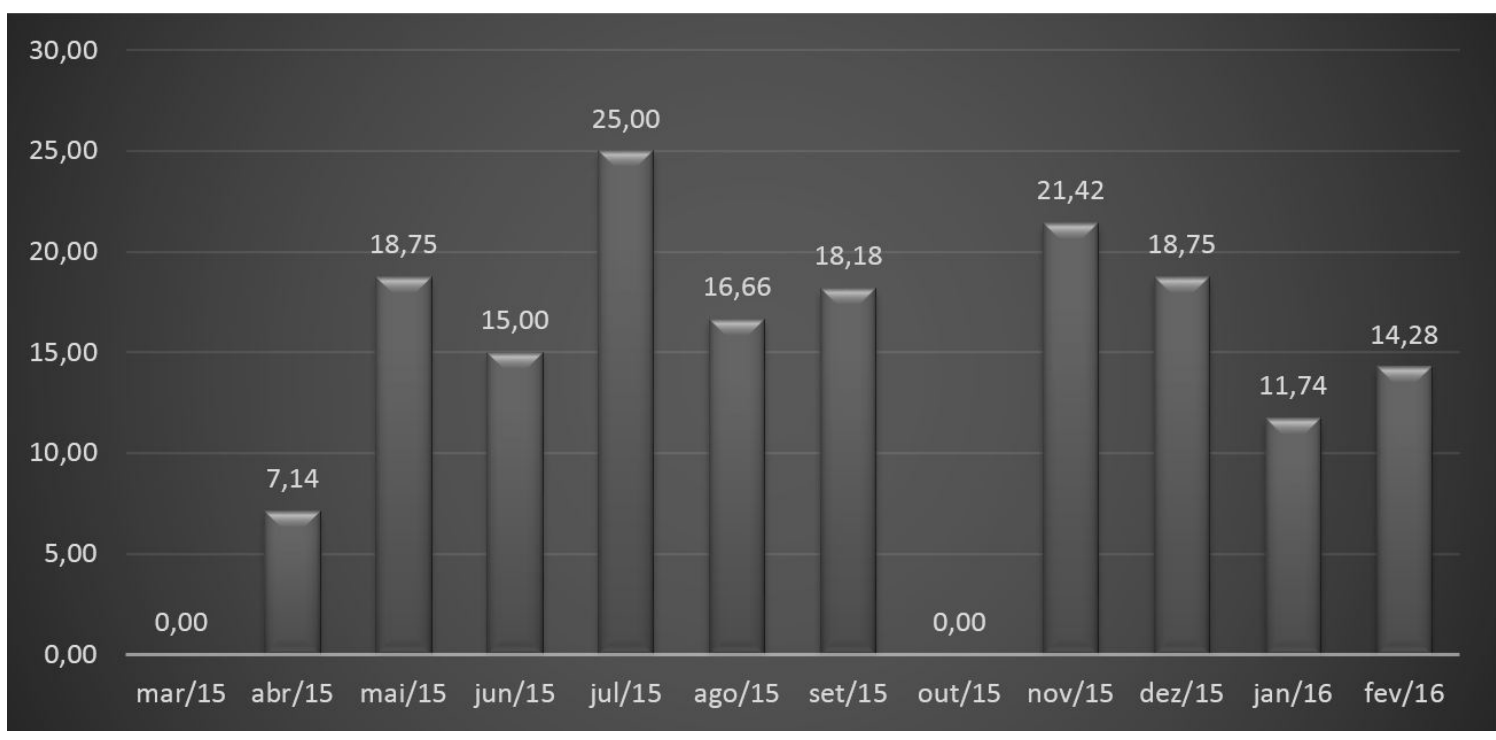

Figura 2. Distribuição dos dados de acordo com a Taxa de Pacientes em Ventilação Mecânica Prolongada na UTI Pediátrica no período de março/2015 a fevereiro/2016. Fortaleza/CE, 2016

\section{DISCUSSÃO}

No presente estudo, a idade média dos pacientes internados na UTI Pediátrica foi de 10,63 $\pm 0,50$ anos, no entanto, essa média ficou acima dos estudos realizados por Hanashiro et al. ${ }^{7}$ que foi de 5,4 $\pm 5,0$ anos, e de Khan et al. ${ }^{8}$ que apresentou uma média de 4,07 $\pm 4,42$ anos. Todavia, as amostras desses estudos abordam patologias diversas e incapacitantes como: doenças neuromusculares, neurológicas e síndromes genéticas, apontando comprometimentos mais precoces, diferentemente da principal causa que acomete o presente público, sendo acometidos principalmente por traumas, sejam eles diretos ou indiretos, tratando-se de um grupo claramente exposto a situações de risco, estabelecendo assim uma diferenciação de causas e idades.

O gênero masculino teve uma prevalência significativa no presente estudo, confirmando os dados de Abelha et al. ${ }^{9}$, que apresentaram $60,5 \%$ do gênero masculino. No entanto, no estudo realizado por Müller et al. ${ }^{10}$ a prevalência foi do gênero feminino, com $55,9 \%$, e crianças portadoras de câncer, diferentemente do que se evidenciou, pois o presente estudo demonstrou maior exposição a situações de risco por parte do gênero masculino.

Analisando-se o diagnóstico decorrente à internação dos pacientes, foi possível detectar que o Traumatismo Crânioencefálico (TCE) predominou como uma das causas de admissão na UTI Pediátrica, tendo uma alta percentual nos meses de maio e julho/15 e fevereiro/16, justificando-se por uma provável associação de fatores, podendo ser entendido como uma soma da hiperatividade infantil com o período de festas carnavalescas por todo o país, e por se tratar do mês de férias da maioria dos pacientes, estando assim sujeitos a fatalidades decorrentes das situações a que as crianças são expostas.
A média percentual da taxa de ocupação no período do estudo foi de $86,13 \%$, estando de acordo com os estudos de Agência Nacional de Saúde Suplementar ${ }^{11}$, pois a taxa de ocupação relatada para a UTI Pediátrica da rede do SUS (Sistema Único de Saúde) foi de $82,4 \%$, com variações de $71,1 \%$ a $89,5 \%$, confirmando os dados do presente estudo.

Por se tratar de uma classe de pacientes em estado crítico, a UTI Pediátrica evidencia um alto índice de internação, e em decorrência dessas causas, a taxa de ocupação é alta, assim como o tempo de permanência. Ressalta-se que no período em estudo os pacientes na Unidade de Terapia Intensiva Pediátrica apresentaram um tempo médio de permanência de $15,52 \pm 0,94$ dias.

De acordo com Lanetzki et al. ${ }^{12}$ em UTI Pediátrica no Estado de São Paulo a média de permanência foi de 9,7 dias em 2009. O Compromisso com a Qualidade Hospitalar ${ }^{13}$ relatou que o tempo médio de permanência na UTI pediátrica é de 7,4 a 9,9 dias, esses valores ficaram bem abaixo do presente estudo, pois a elevação dessa média de permanência pode ser devida ao perfil de pacientes com diagnóstico de TCE.

Os pacientes que se encontram nas UTI normalmente usam um auxílio ventilatório, por apresentarem dificuldades respiratórias. No presente estudo, foi evidenciada uma média de permanência de 8,72 \pm 0,47 dias de utilização da ventilação mecânica, que para o estudo de Moniz et al. ${ }^{14}$ foi baixo, visto que o do referido autor foi de 15 dias em média.

A Taxa de Utilização de Ventilação Mecânica do hospital em estudo apresentou uma média que corresponde a $75,30 \%$, valor esse bem acima do apresentado por Damasceno et al. ${ }^{15}$ que foi de $56,5 \%$ correspondente à região Nordeste, e no Brasil de 51,4\%. Esse aumento na taxa de utilização da Ventilação Mecânica no 
presente estudo se deve ao fato de os pacientes admitidos no hospital em estudo apresentarem traumas acentuados, como TCE.

No período do estudo, a taxa média de sucesso de desmame ficou em $53,10 \pm 3,98 \%$, expressando um indicador positivo para a UTI Pediátrica do referido hospital. Porém, os dados apresentados por Assunção et al. ${ }^{16}$ foram de $79,2 \%$ de sucesso, ficando bem acima do presente estudo. Todavia, poucas literaturas abordaram o desmame de pacientes na UTI Pediátrica.

Entretanto, a taxa de pacientes em Ventilação Mecânica prolongada foi equivalente a $25 \%$ no presente estudo, que utilizaram suporte ventilatório por mais de 21 dias. Esse dado pode ser confirmado por Mota et al. ${ }^{17}$, que foi de $25,3 \%$.

No entanto, Damasceno et al. ${ }^{15}$ apresentaram em seu estudo uma taxa de 55,6\% de pacientes em Ventilação Mecânica Prolongada, expressando uma variabilidade na utilização de suporte mecânico ventilatório, decorrente das distintas causas que acometem os pacientes dos hospitais.

Todavia, este estudo apresenta algumas limitações, pois acredita-se que o perfil e pacientes atendidos nessa UTI em estudo sejam bem diferentes de outras unidades, visto que o público envolve crianças vítimas de fatalidades e com idade muitas vezes próxima da adolescência.

\section{CONCLUSÃO}

Concluiu-se que o gênero masculino teve uma frequência maior na UTI Pediátrica, com idade média de 10,63 \pm 0,50 anos e principal acometimento por Traumatismo Crânio encefálico. Os pacientes apresentaram uma média de 8,72 \pm 0,47 dias de utilização da ventilação mecânica e 75,30\% de taxa de utilização de ventilação mecânica. Apresentou-se um percentual de mais de $50 \%$ de sucesso no desmame de ventilação mecânica, ao contrário da taxa de pacientes em ventilação mecânica prolongada na UTI Pediátrica, que se fez inferior a 30\%. Notou-se que $91 \%$ dos pacientes permaneceram mais de 10 dias na UTI Pediátrica, apresentando uma taxa de ocupação superior a $60 \%$.

No decorrer do estudo, pôde-se perceber uma escassez de literaturas que abordassem os indicadores que se fazem presentes dentro das UTI Pediátricas, consequentemente dificultando as comparações de dados, apresentando-se também, algumas vezes, uma não compatibilidade de públicos.

Os indicadores demonstraram ser um instrumento pontual para cada serviço, pois cada Unidade de Terapia Intensiva Pediátrica apresenta sua peculiaridade, com sua população específica.

\section{REFERÊNCIAS}

1. Gouvêa CSD, Travassos C. Indicadores de segurança do paciente para hospitais de pacientes agudos: revisão sistemática. Cad Saude Publica. 2010;26(6):1061-78. PMid:20657973. http://dx.doi.org/10.1590/S0102$311 \mathrm{X} 2010000600002$.

2. Dias CM, Freitas M, Briz T. Indicadores de saúde: uma visão de saúde pública, com interesse em medicina geral e familiar. Rev Port Clin Geral. 2007;23:439-50.

3. PROQUALIS. Indicadores de segurança [Internet]. Rio de Janeiro; 2015 [citado 2015 maio 23]. Disponível em: http://proqualis.net/indicadoresde-seguran $\% \mathrm{C} 3 \% \mathrm{~A} 7 \mathrm{a}$

4. Pan American Health Organization - PAHO. Cultura de la prevención: un modelo de control para las enfermedades prevenibles por vacunación [Internet]. Washington; 2004 [citado 2015 maio 23]. Disponível em: http:// www.paho.org/Spanish/AD/FCH/IM/GTA16_FinalReport_2004.pdf

5. Institute for Innovation and Improvement - NHS. The Good Indicators Guide: understanding how to use and choose indicators [Internet]. Coventry; 2015 [citado 2015 maio 23]. Disponível em: www.apho.org.uk/resource/ view.aspx?RID $=44584$

6. Brasil. Ministério da Saúde. Agência Nacional de Vigilância Sanitária. Instrução Normativa n. 4, de 24 de fevereiro de 2010. Dispõe sobre indicadores para avaliação de Unidades de Terapia Intensiva. Diário Oficial da União [Internet], Brasília, 25 de fevereiro de 2010 [citado 2015 nov 20]. Disponível em: http://portal.anvisa.gov.br/wps/content/Anvisa+Portal/ Anvisa/Inicio/Servicos+de+Saude/Assunto+de +Interesse/Legislacao/ Unidade+de+Terapia+Intensiva

7. Hanashiro M, Franco AOC, Ferraro AA, Troster EJ. Care alternatives for pediatric chronic mechanical ventilation. J Pediatr. 2011;87(2):145-9. PMid:21503381.

8. Khan MR, Maheshwari PK, Iram S, Haque A, Kayaalp C. Readmission to paediatric intensive care unit: frequency, causes and outcome. J Coll Physicians Surg Pak. 2014;24(3):216-7. PMid:24613123.

9. Abelha FJ, Castro MA, Landeiro NM, Neves AM, Santos CC. Mortalidade e o tempo de internação em uma unidade de terapia intensiva cirúrgica. Rev Bras Anestesiol. 2006;56(1):34-45. PMid:19468548. http://dx.doi. org/10.1590/S0034-70942006000100005.

10. Müller AM, Gazzana MB, Silva DR. Desfecho de pacientes com câncer de pulmão admitidos em unidades de terapia intensiva. Rev Bras Ter Intensiva. 2013;25(1):12-6. PMid:23887754. http://dx.doi.org/10.1590/ S0103-507X2013000100004.

11. Agência Nacional de Saúde Suplementar - ANS. Média de permanência UTI pediátrica. Brasília; 2013. p. 1-5. v. 1.01

12. Lanetzki CS, Oliveira CA, Bass LM, Abramovici S, Troster EJ. The epidemiological profile of pediatric intensive care center at hospital israelita albert einstein. Einstein. 2012;10(1):16-21. PMid:23045820. http://dx.doi. org/10.1590/S1679-45082012000100005.

13. Compromisso com a Qualidade Hospitalar - CQH. Programa Compromisso com a Qualidade Hospitalar [Internet]. São Paulo; 2015 [citado 2015 nov 26]. Disponível em: http://www.cqh.org.br/files/3\%20caderno\%20de\%20 ind\%20baixa-res.pdf

14. Moniz M, Silvestre C, Nunes P, Abadesso C, Matias E, Loureiro H, et al. High-frequency oscillatory ventilation in children: a 10-year experience. J Pediatr. 2013;89(1):48-55. PMid:23544810. http://dx.doi.org/10.1016/j. jped.2013.02.008.

15. Damasceno MPCD, David CMN, Souza PCSP, Chiavone PA, Cardoso LTQ, Amaral JLG, et al. Ventilação mecânica no Brasil: aspectos epidemiológicos. 
Rev Bras Ter Intensiva. 2006;18(3):219-28. PMid:25310434. http://dx.doi. org/10.1590/S0103-507X2006000300002.

16. Assunção MSC, Machado FR, Rosseti HB, Penna HG, Serrão CCA, Silva WG, et al. Avaliação de teste de Tubo T como estratégia inicial de suspensão da ventilacao mecanica. Rev Bras Ter Intensiva. 2006;18(2):121-5. PMid:25316633. http://dx.doi.org/10.1590/S0103-507X2006000200003.
17. Mota EM, Garcia PCR, Piva JP, Fritscher CC. A influência da desnutrição na utilização de ventilação mecânica em crianças admitidas em UTI pediátrica. J Pediatr. 2002;78(2):146-52. PMid:14647797. http://dx.doi. org/10.2223/JPED.825.

Recebido em: Ago. 20, 2016 Aprovado em: Fev. 14, 2017 